This is an electronic reprint of the original article. This reprint may differ from the original in pagination and typographic detail.

\author{
Author(s): Pořízka, Pavel; Kaski, Saara; Hrdlička, Aleš; Škarková, Pavlína; Sládková, Lucia; \\ Häkkänen, Heikki; Prochazka, David; Novotný, Jan; Gadas, Petr; Čelko, Ladislav; \\ Novotný, Karel; Kaiser, Jozef
}

Title: Detection of fluorine using laser-induced breakdown spectroscopy and Raman spectroscopy

Year: $\quad 2017$

Version:

Please cite the original version:

Pořízka, P., Kaski, S., Hrdlička, A., Škarková, P., Sládková, L., Häkkänen, H., Prochazka, D., Novotný, J., Gadas, P., Čelko, L., Novotný, K., \& Kaiser, J. (2017). Detection of fluorine using laser-induced breakdown spectroscopy and Raman spectroscopy. Journal of Analytical Atomic Spectrometry, 32(10), 1966-1974.

https://doi.org/10.1039/C7JA00200A

All material supplied via JYX is protected by copyright and other intellectual property rights, and duplication or sale of all or part of any of the repository collections is not permitted, except that material may be duplicated by you for your research use or educational purposes in electronic or print form. You must obtain permission for any other use. Electronic or print copies may not be offered, whether for sale or otherwise to anyone who is not an authorised user. 

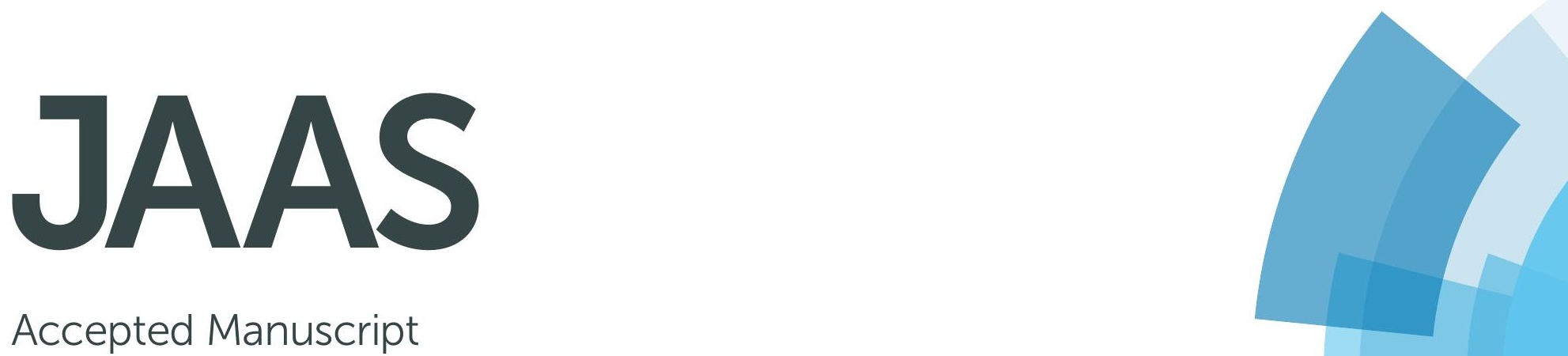

\section{Accepted Manuscript}

This article can be cited before page numbers have been issued, to do this please use: P. Poízka, S. Kaski, A. Hrdlika, P. Škarková, L. Sládková, H. Häkkänen, D. Prochazka, J. Novotný, P. Gadas, L. elko, K. Novotný and J. Kaiser, J. Anal. At. Spectrom., 2017, DOI: 10.1039/C7JA00200A.

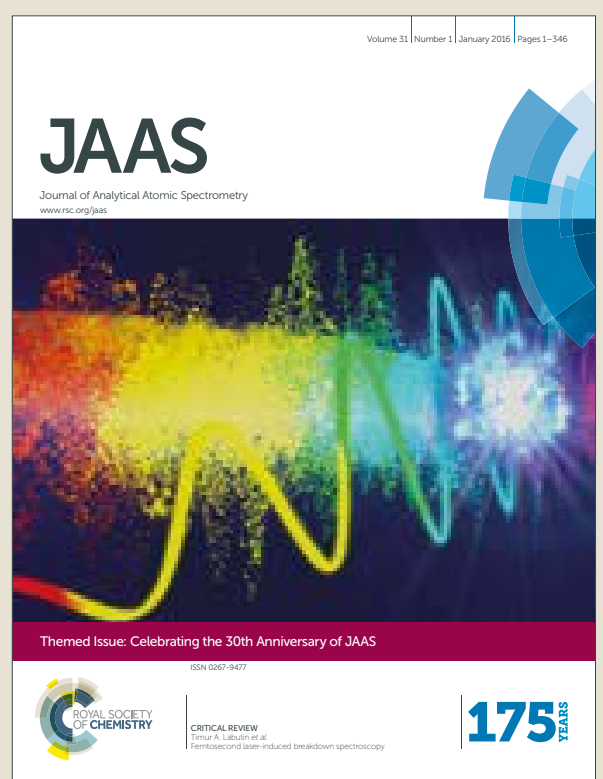

This is an Accepted Manuscript, which has been through the Royal Society of Chemistry peer review process and has been accepted for publication.

Accepted Manuscripts are published online shortly after acceptance, before technical editing, formatting and proof reading. Using this free service, authors can make their results available to the community, in citable form, before we publish the edited article. We will replace this Accepted Manuscript with the edited and formatted Advance Article as soon as it is available.

You can find more information about Accepted Manuscripts in the author guidelines.

Please note that technical editing may introduce minor changes to the text and/or graphics, which may alter content. The journal's standard Terms \& Conditions and the ethical guidelines, outlined in our author and reviewer resource centre, still apply. In no event shall the Royal Society of Chemistry be held responsible for any errors or omissions in this Accepted Manuscript or any consequences arising from the use of any information it contains. 


\title{
Detection of fluorine using Laser-Induced Breakdown Spectroscopy and Raman spectroscopy
}

\author{
Pavel Pořízka, ${ }^{* a}$ Saara Kaski, ${ }^{b}$ Aleš Hrdlička, ${ }^{c}$ Pavlína Modlitbová, ${ }^{a}$ Lucia Sládková, ${ }^{a}$ \\ Heikki Häkkänen, ${ }^{d}$ David Prochazka, ${ }^{a}$ Jan Novotný, ${ }^{a}$ Petr Gadas, ${ }^{e}$ Ladislav Čelko, ${ }^{a}$ \\ Karel Novotný, ${ }^{c}$ and Jozef Kaiser ${ }^{a}$
}

Received Date
Accepted Date

DOI: $10.1039 / x x x x x x x x x x$

www.rsc.org/journalname
In general, the detection of $\mathrm{F}$ and other halogens is challenging for conventional techniques. In this manuscript, various approaches in the qualitative and quantitative analysis of $F$ using LaserInduced Breakdown Spectroscopy (LIBS) technique were demonstrated. In LIBS, fluorine detection can be provided by means of atomic line and molecular bands. For the purposes of our experiment, two sets of pellets with various contents of $\mathrm{CaF}_{2}, \mathrm{CaCO}_{3}$ and cellulose were analyzed using lab-based LIBS system under $\mathrm{He}$ atmosphere. Fluorine atomic line was correlated with $\mathrm{CaF}$ signals proving their close relation. Consequently, limits of detection were determined for both analytical signals. Moreover, conditions necessary for quantification of $\mathrm{F}$ via $\mathrm{CaF}$ band signal were estimated. Dependence of $\mathrm{CaF}$ signal on varying ratio of $\mathrm{Ca}$ and $\mathrm{F}$ content was investigated. Finally, a chip of real $\mathrm{CaF}_{2}$ crystal was prepared and its surface mapped with Raman and LIBS systems. The correlation of obtained elemental and molecular maps showed good numerical correlations. Thus, yielded results validated the possibility to substitute the fluorine atomic line by non-conventional $\mathrm{CaF}$ molecular band in the qualitative and quantitative LIBS analysis of fluorine.

\section{Introduction}

The fluorine element belonging to the group of halogens is widely used in several industrial and scientific domains. Fluorine and its compounds can be found in fire extinguishers, pharmaceuticals ${ }^{1}$, polymers ${ }^{2,3}$, etc. The halogens can be found in a few areas and related issues which have been recently discussed very intensively, e.g. chlorine in concrete ${ }^{4-9}$, fluorine and chlorine in Mars conditions ${ }^{10}$, pharmaceutics ${ }^{11}$, etc.

The common analytical methods used for the determination of fluorine are volumetric analysis, ion selective electrode, ion chromatography, gas chromatography, etc. These types of analysis include sample treatments as dissolution, separation, etc. Other techniques used for direct solid investigation providing multi-

\footnotetext{
${ }^{a}$ CEITEC BUT, Central European Institute of Technology, Brno University of Technology, Purkyňova 656/123, CZ-612 00 Brno, Czech Republic.

${ }^{b}$ Nanoscience Center, Department of Chemistry, University of Jyväskylä, P.O.Box 35, FI-40014 Jyväskylä, Finland.

c CEITEC MU, Central European Institute of Technology, Masaryk University, Kamenice 753/5, CZ-625 00, Brno, Czech Republic.

${ }^{d}$ Nanoscience Center, Department of Biological and Environmental Sciences, University of Jyväskylä, P.O.Box 35, FI-40014 Jyväskylä, Finland.

e Department of Geological Sciences, Faculty of Science, Masaryk University, Kotlárská 2, CZ-611 37 Brno, Czech Republic

*corresponding author: pavel.porizka@ceitec.vutbr.cz
}

elemental analysis are e.g. neutron activation analysis (NAA) and $\mathrm{X}$-ray Fluorescence (XRF). However, these are not very sensitive to the light elements, thus it is advisable to proceed with the measurements in protected facilities ${ }^{12}$. The methods of the inductively coupled plasma optical emission spectrometry and the mass spectrometry (ICP-OES and ICP-MS respectively) deal with the limitations to excite and ionize fluorine because of its high ionization potential $(17.42 \mathrm{eV})$ compared to the argon gas, which is typically used in ICP experiments ${ }^{13}$.

Laser-Induced Breakdown Spectroscopy (LIBS) is another analytical method providing fast determination of halogens ${ }^{14}$. This atomic emission method is based on the use of a pulsed laser that is focused on a small area on a sample surface. The pulsed laser produces a breakdown which results in creating plasma with high temperature. One of the main advantages is a multi-element detection of any material (solid, liquid, gaseous) with spatial resolution in the range of $\mu \mathrm{m}$ and potential to in-situ and even stand-off analysis. To the advantages also belongs sensitivity to light elements (e.g. $\mathrm{Li}, \mathrm{Be}$, etc.), which is difficult for other analytical methods $^{14-16}$.

The detection and quantitative analysis of halogens, especially fluorine, is challenging. The problems in the fluorine detection are namely the high excitation energy of fluorine, absorption of its UV emissions in the air and the moderate limits of detection 
(LODs). The above mentioned drawbacks in the fluorine detection are possible to mitigate by the fluorine analysis under vacuum or ambient atmospheres, by utilization of the spectrometers with detectors which are sensitive in UV and VUV ranges, by utilization of the double pulse (DP) LIBS arrangements (i.e. enhancing the plasma temperature to excite $\mathrm{F}$ transitions) or by the detection of lines in a VIS and a NIR range. The detection of the molecular bands originating from recombination of atomic species in laser-induced plasma has been recently of great interest, namely in the LIBS community.

The most intensive emission lines of halogens can be found in the VUV range, which also places higher demands on the experimental system. The measurement in helium atmosphere ${ }^{12}$ improves the signal-to-noise ratio (SNR) of fluorine atomic emission lines - that allows to reach the LODs in the order of 140 $\mu \mathrm{g} / \mathrm{g}^{17}$. Moreover, the study of Tran et al. ${ }^{18,19}$ showed some improvement also for gaseous and particulate fluorides and even for the solid organic compounds. The role of the internal standardization was presented in the study of fluorine in pharmaceuticals using helium flow ${ }^{1}$. In addition, the combination of the helium atmosphere with reduced-pressure conditions enabled the determination of fluorine in pharmaceutical products in the concentration of 0.03 wt. $\%{ }^{20}$.

There is a possibility to use the molecular bands emission which is an alternative to the detection of fluorine using its atomic emission. Those molecules are formed of fluorine and other elements during the laser-induced plasma (LIP) evolution. For instance, fluorine can combine with calcium, which results in producing CaF molecules. Consequently, these molecules serve as an indirect indicator of fluorine presence. This possibility was firstly mentioned in connection with the arc spectroscopy method ${ }^{21,22}$. Afterwards, this approach was also adopted in LIBS. Gaft et al. ${ }^{23}$ used the molecular band emission for the detection of fluorine. The experiment showed that CaF molecular bands have higher optical emission than atomic emission lines. Subsequently, this approach was used for quantitative analysis in mining ores ${ }^{24}$. Fluorine was detected for the first time in Mars conditions by the ChemCam Instrument on the Curiosity Rover using only CaF molecular bands ${ }^{10}$. The quantitative analysis has been performed also for fluorine traces in solid samples in atmospheric LIBS ${ }^{25}$. Most recently the detection of fluorine using the molecular bands has been investigated also for Ca-free samples, where the Ca solution was sprayed on the surface of the fluorine-containing sample $^{26}$.

This manuscript aims mainly to prove the possibility of using the molecular $\mathrm{CaF}$ bands instead of the emission of atomic fluorine in the detection and quantitative LIBS determination of fluorine. Detected LIP spectral variables (atomic lines and molecular bands emissions) are numerically correlated to prove their relation and inter-connection. Then, the LODs for fluorine are estimated using fluorine atomic line and molecular bands. As a supplement to this study, we explored the influence of $\mathrm{Ca}$ abundance on CaF intensity. Finally, the detection of fluorine with LIBS method is supported with Raman analysis. Both techniques are used for mapping of CaF bearing crystal. Obtained results are compared and correlated proving feasibility to utilize LIBS for the
Table 1 Composition of two sets of pellets prepared for LIBS experiments.

Material abundance in ratio $\quad$ Elemental content / wt.\%

\begin{tabular}{lllllll}
\hline $\mathrm{ID}$ & $\mathrm{CaF}_{2}$ & cellulose & $\mathrm{CaCO}_{3}$ & $\mathrm{~F}$ & $\mathrm{Ca}$ & $\mathrm{CHO}$ \\
\hline \multicolumn{7}{l}{ 1st sample set: calibration set } \\
$\mathrm{C}_{1}$ & 0.01 & 1 & 1 & 0.26 & 20.12 & 79.62 \\
$\mathrm{C}_{2}$ & 0.03 & 1 & 1 & 0.76 & 20.4 & 78.84 \\
$\mathrm{C}_{3}$ & 0.05 & 1 & 1 & 1.25 & 20.68 & 78.07 \\
$\mathrm{C}_{4}$ & 0.1 & 1 & 1 & 2.44 & 21.35 & 76.21 \\
$\mathrm{C}_{5}$ & 0.2 & 1 & 1 & 4.66 & 22.59 & 72.75 \\
$\mathrm{C}_{6}$ & 0.3 & 1 & 1 & 6.69 & 23.73 & 69.58 \\
$\mathrm{C}_{7}$ & 0.4 & 1 & 1 & 8.54 & 24.77 & 66.68 \\
$\mathrm{C}_{8}$ & 0.5 & 1 & 1 & 10.26 & 25.73 & 64.02 \\
\hline 2 nd sample set: Ca-influence set & & & \\
\hline $\mathrm{S}_{1}$ & 1 & 1 & 0.1 & 24.42 & 25.1 & 50.48 \\
$\mathrm{~S}_{2}$ & 1 & 1 & 0.2 & 23.31 & 25.78 & 50.91 \\
$\mathrm{~S}_{3}$ & 1 & 1 & 0.3 & 22.3 & 26.39 & 51.31 \\
$\mathrm{~S}_{4}$ & 1 & 1 & 0.4 & 21.37 & 26.96 & 51.67 \\
$\mathrm{~S}_{5}$ & 1 & 1 & 0.5 & 20.51 & 27.48 & 52.01 \\
& & & & &
\end{tabular}

real-time fluorine detection.

\section{Experimental}

\subsection{Samples}

The sample set consisted of a series of pellets and a chip of a real $\mathrm{CaF}_{2}$ crystal, i.e. fluorite mineral. Pellets were created from $\mathrm{CaF}_{2}$ powder (Moldava, CZ) mixed with $\mathrm{CaCO}_{3}$ (Lach-Ner s.r.o., CZ) and cellulose (Merck KGaA, DE) for robustness. The pellets (12 $\mathrm{mm}$ in diameter and $2 \mathrm{~mm}$ thickness) were homogenized manually in a ceramic mortar and manually pressed with a hydraulic press (17 MPa for 45 seconds). Two sets of pellets were produced, listed in Table 1 . The first set of pellets (referred to as calibration set) was created with $\mathrm{CaCO}_{3}$ and cellulose, with constant ratio content. Due to the calibration and estimation of LODs, the varying $\mathrm{CaF}_{2}$ content was added. The second set of pellets (referred to as Ca-influence set) was made with two constant components - $\mathrm{CaF}_{2}$ and cellulose. The varying $\mathrm{CaCO}_{3}$ content was added to assess the influence of $\mathrm{Ca}$ abundance on the intensity of $\mathrm{CaF}$ band in the LIBS spectrum. The pellets have their IDs that will help to identify them throughout the manuscript, see Table 1. Blank sample was created, having the content of $\mathrm{CaCO}_{3}$ and cellulose in the ratio $1: 1$ with no amount of $\mathrm{CaF}_{2}$.

Moreover, the chip of the real $\mathrm{CaF}_{2}$ crystal was cut and was fixed in epoxy, with its surface polished to a smooth $\mathrm{p}$ lane. This pure $\mathrm{CaF}_{2}$ crystal served for the comparative LIBS and Raman analysis, i.e. mapping of its surface was obtained.

\subsection{Laser-Induced Breakdown Spectroscopy}

LIBS instrument (Sci-Trace including the Interaction chamber, both AtomTrace, CZ) at Central European Institute of Technology was already described in our former publication 27 , which deals with a similar analysis of powder materials pressed into pellets. The system consists of a Q-switched laser source (532 nm, $10 \mathrm{~ns}$; CFR Ultra, Quantel, FR), an echelle spectrometer (F/4, R =6000, 190-1100 nm; Emu-65, Catalina Scientific, US) and an EMCCD detector (250-900 nm; Falcon Blue, Raptor Photonics, IE). In the 
case of this manuscript, pellets were mounted on the stage in the LIBS interaction chamber. The conditions in the chamber, as well as typical LIBS parameters, were varied. The optimization process involved laser energy, gate delay, atmosphere surrounding the sample and its pressure. LIBS system optimization was driven with regards to the highest signal-to-noise ratio (SNR), results of optimization are not shown. Under-pressures of either air or $\mathrm{He}$ led in general to shorter plasma persistence and weaker plasma intensity. Consequently, the interaction chamber was filled always with 1 bar of gas during the optimization (either air or He). It was found that ambient air conditions have disruptive influence on the fluorine detection and thus the He atmosphere is preferred.

The optimized conditions are as follows: $100 \mathrm{~mJ}$ laser pulse energy, 500 ns gate delay and $50 \mu$ s gate width. The system was operated with $3 \mathrm{~Hz}$ repetition rate. Each pellet was measured for 125 times, 25 accumulations in 5 different spots with a 500 $\mu \mathrm{m}$ step between individual spots. The ablated crater size was estimated as $250 \mu \mathrm{m}$. Those measurements were run in the He atmosphere; ambient air was firstly vacuumed (down to $1 \mathrm{mbar}$ pressure) and then the chamber was filled back to 1 bar with He (99.998\% purity; SIAD, CZ).

Observed LIBS data were processed in AtomAnalyzer software (AtomTrace, CZ). Each LIBS spectrum was normalized to its total intensity ${ }^{27}$. Higher number of accumulations helps to overcome some potential fluctuation of the s ystem, f or this reason, no spectra were discarded from the data set ${ }^{28}$. Analytical signal was estimated as a numerical sum of the intensity in the region of spectral line/molecular band. In this manuscript, we will collectively mark the spectral lines and the molecular bands as LIP spectral variables, this refers to $L_{1}-L_{9}$ and $B_{1}$ and $B_{2}$ in Figure 1. The list of LIP spectral variables investigated in this work is given in Table 2. Background was subtracted for each LIP spectral variable individually. The background value was estimated as the mean of a narrow wavelength range in close proximity to spectral line or molecular band, this approach is described in detail in our former work ${ }^{29}$. The obtained intensities of analytical signals were then averaged and further processed.

Numerical correlation showing the mutual dependence of individual LIP spectral variables was estimated as follows. Set of vectors representing the variables was constructed from the measurement of both samples sets. Afterward, vectors were numerically correlated using the Pearson method, which is default algorithm in the cor function implemented in the stats package, CRAN software. The number shows the Pearson correlation coefficient. Estimation of mutual correlations between individual LIP spectral variables is schematically depicted in Appendix.

LODs were estimated from the one-point calibration approach which is recommended by Hahn and Omenetto ${ }^{16}$. LODs were computed as a limit concentration $\left(c_{\mathrm{L}}\right)$ for which the detected intensity is above a threshold. This was estimated utilizing related equation (equation $10 \mathrm{~b}$ in ref. ${ }^{16}$ )

$$
c_{L}=k c_{0} R S D_{B} \frac{X_{B}}{X_{S}}
$$

that can be altered as follows

$$
c_{L}=k c_{0} \frac{S D_{B}}{X_{S}}
$$

where $\mathrm{k}$ is a constant equal to $3, \mathrm{c}_{0}$ is the lowest content of $\mathrm{F}$ in the respective sample set, $\mathrm{RSD}_{\mathrm{B}}$ is relative standard deviation of the background, $\mathrm{X}_{\mathrm{B}}$ and $\mathrm{X}_{\mathrm{S}}$ are average responses due to background and signal respectively, and $\mathrm{SD}_{\mathrm{B}}$ is standard deviation of background.

Finally, the $\mathrm{CaF}_{2}$ chip surface was mapped utilizing LIBS system. Due to the uniqueness of the $\mathrm{CaF}_{2}$ chip, no optimization of LIBS system parameters was done prior its analysis. We set the same parameters as in the case of pellets measurements (laser pulse energy and gate delay). One laser pulse was introduced to each spot and LIP spectrum was detected for consecutive analysis, no accumulation or averaging was used. Then, we marked the region of interest, where the variation in $\mathrm{CaF}_{2}$ distribution was seemingly highest. This region was mapped firstly with Raman (settings find $\mathrm{b}$ elow) a nd a fterwards $\mathrm{w}$ ith L IBS $(50 \times 40$ points with $350 \mu \mathrm{m}$ resolution) system. Obtained LIBS map was processed and only the intensities of Ca I $422.67 \mathrm{~nm}$, F I $685.6 \mathrm{~nm}$ and $\mathrm{CaF}$ around $600 \mathrm{~nm}$ were determined in the same manner as described above. Those analytical signals were then correlated with the Raman data.

\subsection{Raman Spectroscopy}

The Raman measurements were carried out at the University of Jyväskylä with home-built Raman setup in the backscattering geometry. The continuous wave laser beam $(532 \mathrm{~nm}$ solid-state laser, $200 \mathrm{~mW}$ laser power; MLL532, CNI, CN) was focused on the sample with an achromatic lens with a focal distance of $30 \mathrm{~mm}$. The Raman scattering was focused to the CCD camera (Newton, Andor, IE) and imaging spectrograph (Czerny-Turner configuration, $\mathrm{f}=500 \mathrm{~mm}$; Acton SP2500i, Princeton Instruments, US) with an entrance slit of $50 \mu \mathrm{m}$ and grating of 600 grooves $/ \mathrm{mm}$. A spectrum was collected at each measurement point using 0.8 ms data accumulation time. The dark noise was subtracted from all the detected data.

The map of real $\mathrm{CaF}_{2}$ crystal was constructed from $60 \times 60$ points with $200 \mu \mathrm{m}$ steps. We used MATLAB (R2015a) software to process the detected spectra. Intensity of $\mathrm{CaF}_{2}$ Raman peak of $323 \mathrm{~cm}^{-1}$ was determined and then corrected for background. Obtained intensities were then correlated with LIBS data.

\section{Results and Discussion}

\subsection{Investigation of LIBS data}

Prior the LIBS analysis itself, extensive optimization of several parameters (laser energy, gate delay, type of ambient atmosphere and its pressure) was provided. However, it is not shown for the sake of briefness. It can be summed up, that in general the lower or reduced pressures has negative effect on the LIP intensity. With lower pressure, the natural containment of LIP by ambient atmosphere is weak which allows the plasma plume to expand to bigger volume and thus to lose considerably more energy. On the other hand, the He atmosphere is beneficial for the excitation of 
energetically demanding species within LIP, for instance the case of fluorine. For those purposes, the He atmosphere of 1 bar was found as the most convenient for laser ablation and detection of fluorine. We are aware that those conditions cannot be met in real life, in-situ applications, but they are perfect for making our point throughout this manuscript, where fluorine atomic line and molecular bands are correlated.

First of all, we thoroughly checked the typical LIBS spectrum that we had obtained from measurement of a sample $S_{5}$ with the highest abundance of F, see Figure 1. LIBS spectrum is dominated by $\mathrm{Ca}$ lines across the whole spectral range. There was only one fluorine line found in the observed wavelength $r$ ange, namely $\mathrm{F}$ I $685.6 \mathrm{~nm}$, which is the most prominent fluorine 1 ine i $\mathrm{n}$ visible range. Two CaF molecular bands were identified in the LIBS spectrum around $530 \mathrm{~nm}$ and $600 \mathrm{~nm}$. All selected LIP spectral variables (atomic and ionic lines, and both $\mathrm{CaF}$ bands) were localized and marked in Figure 1. Zoomed spectral regions around selected F I $685.6 \mathrm{~nm}$ line and CaF molecular bands are depicted in Figure 2. Signal from a LIP of a blank was added to this figure to check the influence of any potential $\mathrm{CaO}$ molecular bands that can interfere with $\mathrm{CaF}$ bands. As it may be seen from the the figure, the lack of fluorine in the sample leads to the vanishing of fluorine related spectral features. It has to be stressed that there seems to be a higher background in the region of orange CaF system (around $600 \mathrm{~nm}$ ). This may be possible related to the presence of $\mathrm{CaO}$ band emission. Nevertheless, such spectral interference do not significantly affect the CaF orange band intensity as was proved by the correlation of spectral intensities.

It is noteworthy that spectral lines in the region of the green CaF system (around $530 \mathrm{~nm}$ ) belong to Ca. According to the NIST database ${ }^{30}$ the lines belong to Ca III $532.13 \mathrm{~nm}$ and Ca I $534.95 \mathrm{~nm}$. Despite the good fit of the central wavelength of the Ca III line with the database, its presence in the LIP spectrum is doubtful. Because, in general more higher ionization levels do not occur in the LIP for its relatively lower temperature. Moreover, this spectral line can be caused by the persistence of the laser light bouncing within the interaction chamber. As in the aforementioned case, the presence of Ca lines do not significantly affect the CaF signal.

A list of selected spectral lines with their quantum properties is given in Table 2. Such a spectrum (detected with $50 \mu$ s gate width) reflects c omplete t emporal a verage of L IP. I t i s a wellknown fact that the ionic lines dominate the LIP emission at the beginning, the atomic lines are usually present afterwards and the molecular bands occur at the end of LIP persistence.

\subsection{LIP spectral variables correlation}

In the next step of the analysis, a calibration set, $\mathrm{C}_{1}-\mathrm{C}_{8}$ listed in Table 1 , was analyzed using LIBS system at given experimental conditions. This sample set was chosen for its high span of $\mathrm{F}$ abundance, while the abundances of $\mathrm{Ca}$ and $\mathrm{F}$ are rising together. Thus, it enables to study relationships between selected LIP spectral variables and consequent changes in their spectral emission. After the LIBS analysis, the intensities of selected LIP spectral variables were calculated as described above. The yielded intensi-

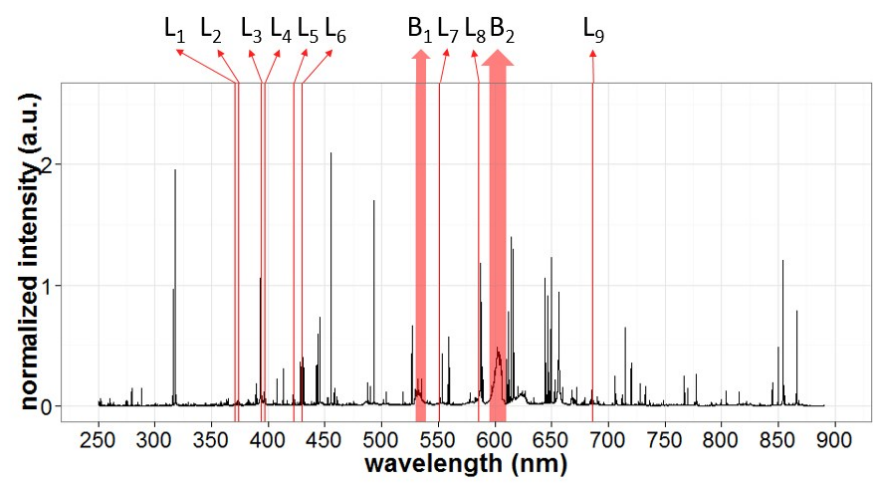

Fig. 1 Typical LIBS spectrum depicted as numerical average of spectra obtained from LIBS measurement of a sample with highest abundance of $\mathrm{Ca}$ and $F$. Spectral lines are assigned as $L_{1}$ to $L_{9}$, see Table 2, and molecular bands as $\mathrm{B}_{1}$ for $\mathrm{CaF}$ around $530 \mathrm{~nm}$ and $\mathrm{B}_{2}$ for $\mathrm{CaF}$ around $600 \mathrm{~nm}$.

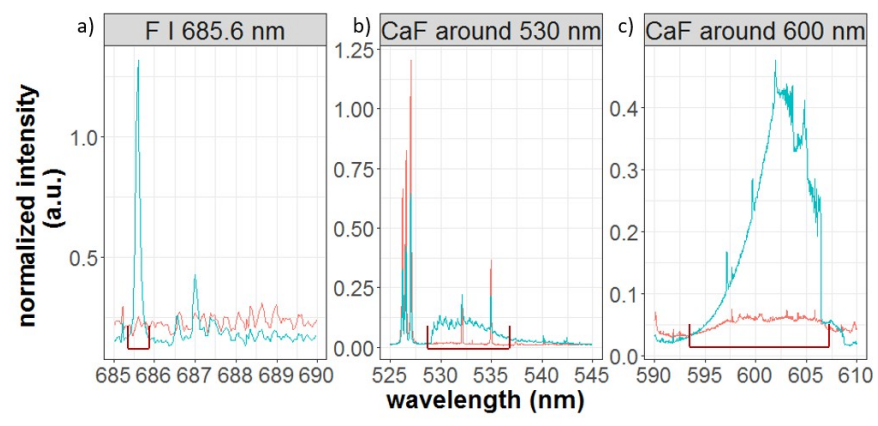

Fig. 2 Enlarged areas of fluorine line and molecular bands in typical LIBS spectra of $\mathrm{CaF}$ containing sample. The highlighted area shows the range from which the numerical integral was computed and considered as the intensity of respective LIP spectral variable. The spectrum depicted in cyan was taken from sample $\mathrm{S} 1$, the pink spectrum is related to the blank sample.

ties were organized into a matrix where rows indicate individual samples and columns indicate averaged intensities of respective variables. Column-wise correlation of pairs of variables was then applied to this data matrix. This correlation approach is schematically depicted in the Appendix. The result is represented by a diagonal correlation matrix, depicted in Figure 3a, where each cell shows the correlation coefficient between selected variables (i.e. LIP spectral variables). Cells on the diagonal are naturally equal to unity.

Division of the correlation matrix, Figure 3a, according to correlation factor into three groups ( $\mathrm{F}$ and $\mathrm{CaF}$ group, $\mathrm{Ca} I$ lines group and Ca II lines group) is evident. In the first group, F I $685.6 \mathrm{~nm}$ line is clustered together with CaF bands ( $\mathrm{L}_{9}$, and $\mathrm{B}_{1}$ and $\mathrm{B}_{2}$ ). This insinuates that $\mathrm{CaF}$ band response follows the abundance of fluorine in the sample. Such behaviour is expectable from the sample's composition. Moreover, those results give evidence of a correct selection of both $\mathrm{CaF}$ band regions. Considering high mutual correlation between F I $685.6 \mathrm{~nm}$ and CaF molecular band responses, it is feasible to substitute a fluorine line by a $\mathrm{CaF}$ molecular band in qualitative and even in quantitative analysis of fluorine in sample of interest. The negative correlation between Ca lines $\left(\mathrm{L}_{1}-\mathrm{L}_{8}\right)$ and F I 685.6 line together with $\mathrm{CaF}$ 
Table 2 List of selected lines and their quantum properties, where $E_{i}$ is lower excitation energy, $E_{j}$ is upper excitation energy, $E_{\text {ion }}$ is ionization energy of the element and $A_{k i}$ is Einstein coefficient. Line ID is also given and used when appropriate. ID of molecular bands is as follows: $B_{1}$ - CaF around $530 \mathrm{~nm}$ and $B_{2}-\mathrm{CaF}$ around $600 \mathrm{~nm}$. Spectral lines were obtained from the NIST database ${ }^{30}$. More information about the molecular bands can be found in the work by Alvarez-Llamas et al. ${ }^{25}$

\begin{tabular}{llllll} 
line & central wavelength $/ \mathrm{nm}$ & $\mathrm{E}_{\mathrm{i}}\left(\mathrm{E}_{\mathrm{i}}+\mathrm{E}_{\mathrm{ion}}\right) / \mathrm{eV}$ & $\mathrm{E}_{\mathrm{j}}\left(\mathrm{E}_{\mathrm{j}}+\mathrm{E}_{\text {ion }}\right) / \mathrm{eV}$ & $\mathrm{A}_{\mathrm{ki}} / .108 \mathrm{~s}^{-1}$ & $\mathrm{ID}$ \\
\hline Ca II & 370.60 & $3.12(9.23)$ & $6.47(12.58)$ & 0.88 & $\mathrm{~L}_{1}$ \\
Ca II & 373.69 & $3.15(9.26)$ & $6.47(12.58)$ & 1.70 & $\mathrm{~L}_{2}$ \\
Ca II & 393.37 & $0.00(6.11)$ & $3.15(9.26)$ & 1.47 & $\mathrm{~L}_{3}$ \\
Ca II & 396.85 & $0.00(6.11)$ & $3.12(9.23)$ & 1.40 & $\mathrm{~L}_{4}$ \\
Ca I & 422.67 & 0.00 & 2.93 & 2.18 & $\mathrm{~L}_{5}$ \\
Ca I & 430.25 & 1.89 & 4.78 & 1.38 & $\mathrm{~L}_{6}$ \\
Ca I & 551.30 & 2.93 & 5.18 & 1.10 & $\mathrm{~L}_{7}$ \\
Ca I & 585.75 & 2.93 & 5.04 & 0.66 & $\mathrm{~L}_{8}$ \\
F I & 685.60 & 12.73 & 14.54 & 0.24 & $\mathrm{~L}_{9}$
\end{tabular}

molecular emission bands is particulary noteworthy. However, this negative correlation is still not fully described.

Mutual correlation between spectral variables can uncover their relation or independence. High correlation between ionic Ca lines $\left(\mathrm{L}_{1}-\mathrm{L}_{4}\right)$ suggests the possibility to randomly pick up any of selected lines to fully represent the whole group. This assumption is, of course, valid for atomic Ca lines $\left(\mathrm{L}_{5}-\mathrm{L}_{8}\right)$. However, the intra-correlation of both sets of Ca lines (atomic vs. ionic) is slightly dependent on their quantum properties, Table 2 . Lower correlation between ionic and atomic Ca lines is also evident. Summarizing, the more similar the higher energy level of compared spectral lines, the higher the mutual correlation.

The selection of the spectral range from which the molecular band intensity is determined was also investigated using the correlation matrix, data not shown. This was done in accordance with the theory where each part of spectral line is fully correlated with the rest of the line. Thus, the both investigated molecular bands showed good correlation between each other and with the fluorine atomic line. They showed it in their whole spectral range as well as in any spectral section from either the green (around $530 \mathrm{~nm}$ ) or the orange (around $600 \mathrm{~nm}$ ) system. This knowledge could be crucial for the use of the orange $\mathrm{CaF}$ system which can be partially overlapped with the $\mathrm{CaO}$ band yet fortunately that is not our case, Figure 2.

\subsection{Influence of Ca content}

The second sample set, $S_{1}-S_{5}$ in Table 1 , was measured in order to study the influence of varying Ca content in the sample set on the intensity of $\mathrm{CaF}$ band. In this sample set, fluorine content was decreasing and calcium content was increasing. Responses of selected LIP spectral variables were investigated using the correlation matrix in the same manner as in the previous case. In Figure $3 \mathrm{~b}$, the correlation matrix fell apart leaving no inter-correlated LIP spectral variables. High intra-correlation of atomic and ionic Ca lines $\left(\mathrm{L}_{5}-\mathrm{L}_{8}\right.$ and $\mathrm{L}_{1}-\mathrm{L}_{4}$ respectively) was present. But the inter-correlation of atomic and ionic Ca species was low. Fluorine was anticorrelated to Ca I emissions, which is obvious from composition of samples, and there was low anticorrelation with Ca II emissions. However, the most important phenomenon was no correlation between F I $685.6 \mathrm{~nm}$ line $\left(\mathrm{L}_{9}\right)$ and CaF molecular responses $\left(B_{1}\right.$ and $\left.B_{2}\right)$.
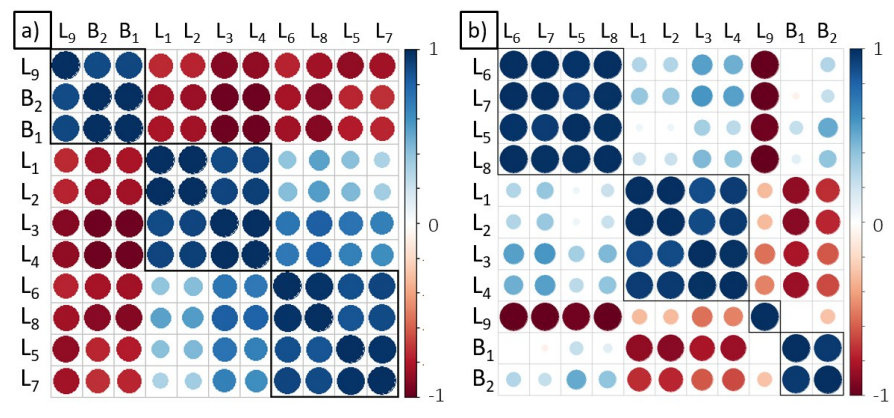

Fig. 3 a) Correlation matrix (diagonal matrix) of selected spectral lines and molecular bands intensities, when calibration set of samples $\left(C_{1}\right.$ $\mathrm{C}_{8}$ ) was measured. b) Correlation matrix (diagonal matrix) of selected LIP spectral variables intensities, when $\mathrm{Ca}$-influence set of samples was measured $\left(S_{1}-S_{5}\right)$. Blue color show high correlation between two variables, red color uncovers negative correlation between two variables. Spectral lines are assigned with $L_{1}$ to $L_{9}$ and can be found in Table 2, molecular bands are assigned as follows: $\mathrm{B}_{1}-\mathrm{CaF}$ band around $530 \mathrm{~nm}$ and $\mathrm{B}_{2}-\mathrm{CaF}$ band around $600 \mathrm{~nm}$.

Presented results suggest that CaF signal should not be used as a mean in the detection of $F$, especially in the sample set where the changes in fluorine content and its related intensity are not directly correlated with the changes in CaF band intensity. This phenomenon could be explained by the variations in $\mathrm{CaF}$ and $\mathrm{F}$ signals where, depending on relatively high $\mathrm{Ca}$ and $\mathrm{F}$ contents in the sample, various influence from self-absorption effect could be present. Another possible explanation may be seen in the $\mathrm{Ca}: \mathrm{F}$ molar ratio. In other words, considerably higher content of Ca to $\mathrm{F}$ has positive effect on the formation of $\mathrm{CaF}$ bands. However, the dynamics of $\mathrm{CaF}$ bands formation in the LIP are not favorable for similar $\mathrm{Ca}$ and $\mathrm{F}$ contents, low molar ratios. Such evidence leads to the conclusion also presented in the work by Alvarez-Llamas et al. ${ }^{25}$ In their work they suggested Ca:F molar ratios to be over 20:1 in order to avoid any disturbances in CaF signal caused by insufficient Ca content.

\subsection{Calibration}

Calibration curves for fluorine were constructed based on F I $685.6 \mathrm{~nm}$ and $\mathrm{CaF}$ around $600 \mathrm{~nm}$ intensity responses, in Figure 4 . The trends in both calibration plots differ, regardless of the results shown in the correlation matrix, Figure 3. In both cases, 


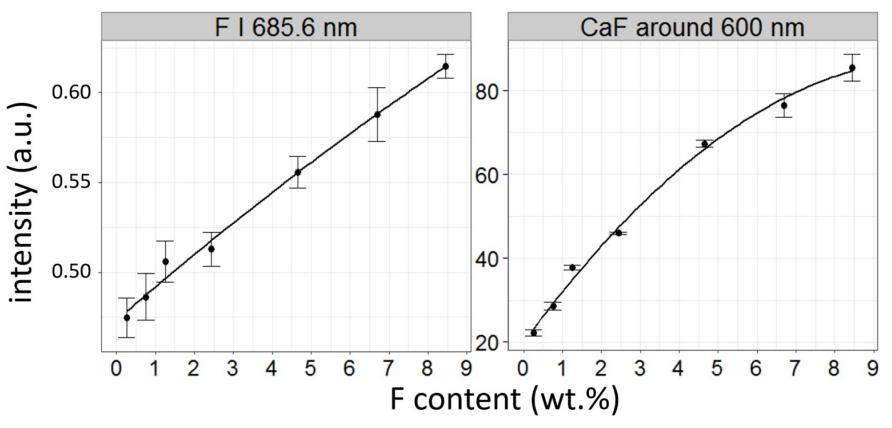

Fig. 4 Calibration curves for F I $685.6 \mathrm{~nm}$ line and molecular band of $\mathrm{CaF}$ around $600 \mathrm{~nm}$.

Table 3 Limits of detection estimated for F I $685.6 \mathrm{~nm}$ and $\mathrm{CaF}$ bands.

\begin{tabular}{ll} 
LIP spectral variable & LOD / ppm \\
\hline F I $685.6 \mathrm{~nm}$ & 150 \\
CaF around $600 \mathrm{~nm}$ & 65 \\
CaF around $530 \mathrm{~nm}$ & 120
\end{tabular}

the curve rises steeply for low concentrations. but in the case of $\mathrm{CaF}$ system it rises linearly only up to circa $2 \mathrm{wt} . \%$ of $\mathrm{F}$ content, then it levels off. The slope of the calibration curve is steeper for $\mathrm{CaF}$ band suggesting lower LODs. Relative standard deviation of either $\mathrm{F}$ or $\mathrm{CaF}$ signal varies from 2 to $7 \%$ regardless of the sample composition.

The resulting plots of the F I line and the CaF band show that the self-absorption phenomena manifest itself more strongly in the case of the $\mathrm{CaF}$ band. It may be supposed that $\mathrm{CaF}$ radicals are more concentrated and less excited in the peripheral parts of the laser-induced plasma than fluorine a toms. This is due to the fact that fluorine require higher temperature closer to the hot core of the microplasma ${ }^{23}$.

The LODs were estimated from the measurement of sample $\mathrm{C}_{1}$, containing $0.26 \mathrm{wt} . \%$ of $\mathrm{F}$ and $20.12 \mathrm{wt} . \%$ of $\mathrm{Ca}$. The LODs of $\mathrm{F}$ were estimated from the signal of F I $685.6 \mathrm{~nm}$ line and $\mathrm{CaF}$ emission bands, Table 3. Computation of LODs of F using its atomic line and related molecular bands is supported by above mentioned high mutual correlation of F I $685.6 \mathrm{~nm}$ signal with $\mathrm{CaF}$ bands. Lower LODs were observed in the case of CaF bands, this is a result of higher intensity due to easier excitation of $\mathrm{CaF}$ bands. This suggests higher emission intensity of molecular bands over atomic transitions in He atmosphere. This fact is in agreement with already published work ${ }^{23}$, where the LIBS analysis was done directly in air.

\subsection{LIBS and Raman mapping}

All of the Raman spectra, which were measured from a violet colored fluorite-bearing c rystal, contained the $323 \mathrm{c} \mathrm{m}^{-1}$ peak of $\mathrm{CaF}_{2}$, which indicates that there is no change in the mineral structure. However, we observed some small side peaks, especially in the middle of the crystal. The strongest from those side peaks was one with the value of around $420 \mathrm{~cm}^{-1}$, see Figure 5. The intensity of such side peaks might depend on the violet color saturation ${ }^{31}$. Moreover, this intensity might be caused by the radiation-induced

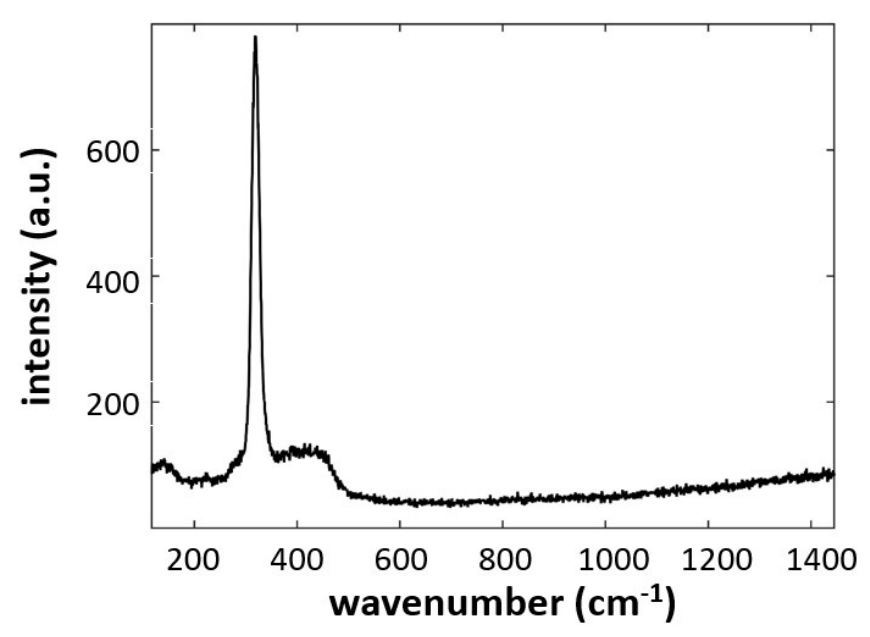

Fig. 5 Raman spectrum of natural fluorite sample measured from the middle of the violet crystal shows strong $\mathrm{CaF}_{2}$ peak at $323 \mathrm{~cm}^{-1}$.

damage in the crystal structure ${ }^{32}$. In some spectra, there was also some broad fluorescence above $500 \mathrm{~cm}^{-1}$, origin unknown.

To overlap LIBS maps with the Raman map, following data processing steps were undertaken. First, obtained spectra were processed and intensities of Ca I $422 \mathrm{~nm}$, F I $685.6 \mathrm{~nm}$ and $\mathrm{CaF}$ molecular band around $600 \mathrm{~nm}$ were determined together with the $323 \mathrm{~cm}^{-1}$ Raman peak of $\mathrm{CaF}_{2}$. As there was no sign of other Raman peaks, it could be assumed, that the crystal was fluorite with minimal impurities. The slight intensity variation in the Raman map is most likely due to the laser power fluctuation or some absorbance on the sample surface. In the mineral analysis, however, there are multiple factors contributing to the scattering intensity, e.g. the crystal orientation or crystal defects ${ }^{33}$.

Second, LIBS and Raman maps were individually scaled to $[0,1]$ in their full range and then cropped to cover the same area. Then, to obtain the same number of pixels in each image $(60 \times 30$ pixels), the original low-resolution LIBS maps were refined and the values interpolated. The elemental and molecular maps were plotted in B\&W scale, see Figure 6. All maps (LIBS and Raman) were then numerically correlated, Table 4. Mutual correlations of selected analytical signals plotted in the LIBS maps and in the Raman map are relatively high and support the possibility to utilize any of selected LIBS analytical signals ( $\mathrm{F}$ atomic line or CaF bands) to fully describe the distribution of fluorine on the sample surface. The LIBS map of Ca I $422.67 \mathrm{~nm}$ is also depicted and shows good correlation with other signals, suggesting possible use of $\mathrm{CaF}$ for quantitative analysis of $\mathrm{F}$ in the sample.

Table 4 correlation coefficients of Raman and LIBS maps depicted in Figure 6.

\begin{tabular}{lll}
\multicolumn{2}{l}{ correlation pairs } & level of correlation \\
\hline \multirow{2}{*}{ Raman signal } & Ca I & 0.63 \\
& F I & 0.73 \\
& CaF & 0.78 \\
\hline \multirow{2}{*}{ Ca I } & F I & 0.9 \\
& CaF band & 0.68 \\
\hline F I & CaF band & 0.82
\end{tabular}




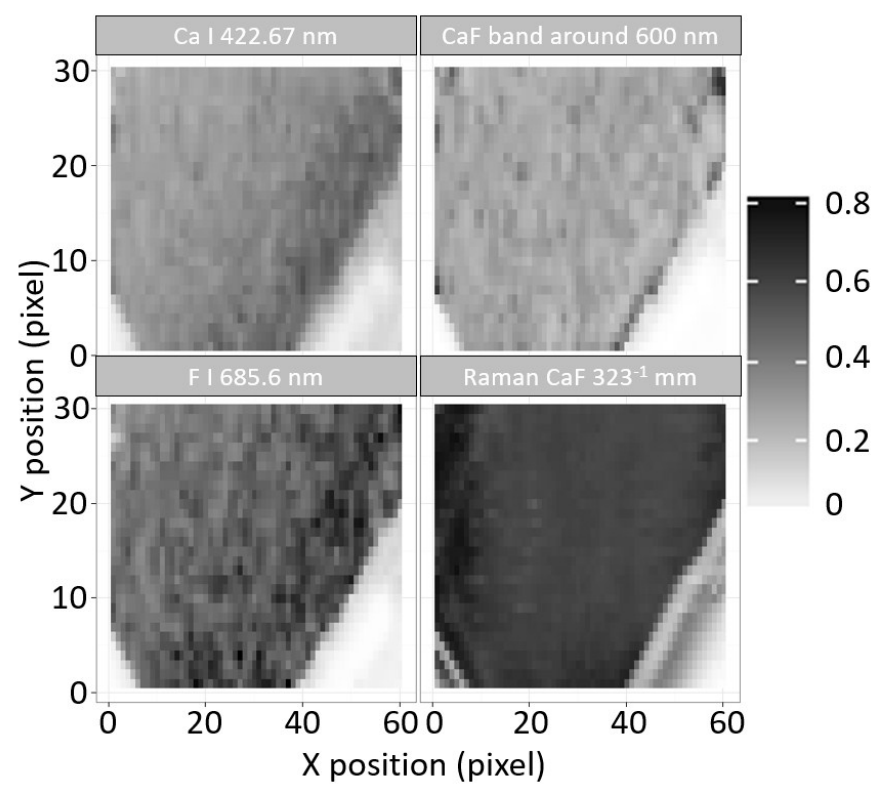

Fig. 6 LIBS maps of Ca I $422.67 \mathrm{~nm}$ (Ca line), F I $685.6 \mathrm{~nm}$ (F line) and $\mathrm{CaF}$ band around $600 \mathrm{~nm}$ and Raman signal ( $\mathrm{CaF}_{2}$ band) map depicting the surface elemental and molecular analyses of $\mathrm{CaF}_{2}$ crystal fixed in epoxy.

\section{Conclusions}

The challenges in detection of fluorine can be overcome by utilization of LIBS technique. For that reason, LIBS spectrum of $\mathrm{CaF}_{2}$ containing pellet was thoroughly investigated. Along the number of $\mathrm{Ca}$ atomic and ionic lines, LIP spectral variables representing the $\mathrm{F}$ content ( $\mathrm{F}$ atomic line and also $\mathrm{CaF}$ molecular bands) were observed. Further data processing (numerical correlation) of intensities detected during the LIBS analysis showed a close relation of LIP spectral variables (F, Ca and even CaF signals). Their correlation coefficient is however strongly dependent on the ratio of Ca:F contents. Random changes in Ca:F contents between samples/measurement spots have disruptive effect on the correlation of $\mathrm{Ca}, \mathrm{F}$ and $\mathrm{CaF}$ signals. Considering only the calibration samples set in which the $\mathrm{Ca}$ and $\mathrm{F}$ contents are rising together, high correlation of all LIP spectral variables was discovered. Based on these results, necessary conditions were constructed to support the utilization of $\mathrm{CaF}$ bands in quantitative analysis of fluorine in the sample containing $\mathrm{Ca}$ and $\mathrm{F}$.

The Raman spectroscopy was employed as a reference to LIBS technique in order to prove the possibility of representing the $\mathrm{F}$ content via means of $\mathrm{CaF}$ signal. $\mathrm{A} \mathrm{CaF}_{2}$ crystal was chipped and its surface mapped using consecutively Raman spectroscopy and LIBS. The molecular $\left(\mathrm{CaF}_{2}\right.$ Raman signal) and elemental (Ca I $422.67 \mathrm{~nm}$, F I $685.6 \mathrm{~nm}$ and CaF band around $600 \mathrm{~nm}$ ) maps were numerically correlated. Obtained results (solely from LIBS and from LIBS and Raman tandem analyses) supported the utilization of $\mathrm{CaF}$ band signal in detection of $\mathrm{F}$ in mineral sample.

\section{Acknowledgement}

We would like to acknowledge that this research was carried out with financial support from the Ministry of Education, Youth and
Sports of the Czech Republic under the National Sustainability Programme II, the project CEITEC 2020 (grant LQ1601). This work was carried out with the support of CEITEC Nano Research Infrastructure (grant LM2015041; MEYS CR), CEITEC Brno University of Technology. SK and $\mathrm{HH}$ would like to acknowledge the support of the research by the Academy of Finland (grant 292558).

\section{Conflict of interest}

There are no conflicts of interest to declare.

\section{References}

1 L. St-Onge, V. Detalle and M. Sabsabi, Spectrochimica Acta Part B: Atomic Spectroscopy, 2002, 57, 121-135.

2 R. Sattmann, I. Monch, H. Krause, R. Noll, S. Couris, A. Hatziapostolou, A. Mavromanolakis, C. Fotakis, E. Larrauri and R. , Applied Spectroscopy, 1998, 52, 456-461.

3 E. D. Lancaster, K. L. McNesby, R. G. Daniel and A. W. Miziolek, Applied Optics, 1999, 38, 1476-1480.

4 G. Wilsch, F. Weritz, D. Schaurich and H. Wiggenhauser, Construction and Building Materials, 2005, 19, 724-730.

5 C. D. Gehlen, E. Wiens, R. Noll, G. Wilsch and K. Reichling, Spectrochimica Acta Part B: Atomic Spectroscopy, 2009, 64, 1135-1140.

6 K. Sugiyama, T. Fujii, T. Matsumura, Y. Shiogama, M. Yamaguchi and K. Nemoto, Applied Optics, 2010, 49, 181-190.

7 M. A. Gondal, M. A. Dastageer, M. Maslehuddin, A. J. Alnehmi and O. S. B. Al-Amoudi, Applied Optics, 2011, 50, 3488-3496.

8 T. A. Labutin, A. M. Popov, S. N. Raikov, S. M. Zaytsev, N. A. Labutina and N. B. Zorov, Journal of Applied Spectroscopy, 2013, 80, 315-318.

9 T. A. Labutin, A. M. Popov, S. M. Zaytsev, N. B. Zorov, M. V. Belkov, V. V. Kiris and S. N. Raikov, Spectrochimica Acta Part B: Atomic Spectroscopy, 2014, 99, 94-100.

10 O. Forni, M. Gaft, M. J. Toplis, S. M. Clegg, S. Maurice, R. C. Wiens, N. Mangold, O. Gasnault, V. Sautter, S. Le Mouelic and et al., Geophysical Research Letters, 2015, 42, 1020-1028.

11 W. R. Dolbier, Journal of Fluorine Chemistry, 2005, 126, 157163.

12 D. A. Cremers and L. J. Radziemski, Analytical Chemistry, 1983, 55, 1252-1256.

13 P. A. Mello, J. S. Barin, F. A. Duarte, C. A. Bizzi, L. O. Diehl, E. I. Muller and E. M. M. Flores, Analytical and Bioanalytical Chemistry, 2013, 405, 7615-7642.

14 D. A. Cremers and L. J. Radziemski, Handbook of LaserInduced Breakdown Spectroscopy, John Wiley, 2006.

15 D. W. Hahn and N. Omenetto, Applied Spectroscopy, 2010, 64, 335-366.

16 D. W. Hahn and N. Omenetto, Applied Spectroscopy, 2012, 66, 347-419.

17 C. D. Quarles, J. J. Gonzalez, L. J. East, J. H. Yoo, M. Morey and R. E. Russo, Journal of Analytical Atomic Spectrometry, 2014, 29, 1238-1242. 
18 M. Tran, Q. Sun, B. W. Smith and J. D. Winefordner, Applied Spectroscopy, 2001, 55, 739-744.

19 M. Tran, B. W. Smith, D. W. Hahn and J. D. Winefordner, Applied Spectroscopy, 2001, 55, 1455-1461.

20 G. Asimellis, S. Hamilton, A. Giannoudakos and M. Kompitsas, Spectrochimica Acta Part B: Atomic Spectroscopy, 2005, 60, 1132-1139.

21 J. Papish, L. E. Hoag and W. E. Snee, Industrial, 1930, 2, 263264.

22 O. B. Troshkina, Journal of Applied Spectroscopy, 1971, 14, 430-437.

23 M. Gaft, L. Nagli, N. Eliezer, Y. Groisman and O. Forni, Spectrochimica Acta Part B: Atomic Spectroscopy, 2014, 98, 39-47.

24 C. Alvarez, J. Pisonero and N. Bordel, Spectrochimica Acta Part B: Atomic Spectroscopy, 2014, 100, 123-128.

25 C. Alvarez-Llamas, J. Pisonero and N. Bordel, Spectrochimica Acta Part B: Atomic Spectroscopy, 2016, 123, 157-162.

26 C. Alvarez-Llamas, J. Pisonero and N. Bordel, J. Anal. At. Spectrom, 2017, 32, 162-166.

27 P. Porizka, J. Klus, A. Hrdlicka, J. Vrabel, P. Skarkova, D. Prochazka, J. Novotny, K. Novotny and J. Kaiser, Journal of Analytical Atomic Spectrometry, 2017, 32, 277-288.

28 P. Porizka, J. Klus, D. Prochazka, E. Kepes, A. Hrdlicka, J. Novotny, K. Novotny and J. Kaiser, Spectrochimica Acta Part B: Atomic Spectroscopy, 2016, 123, 114-120.

29 D. Prochazka, M. Bilik, P. Prochazkova, J. Klus, P. Porizka, J. Novotny, K. Novotny, B. Ticova, A. Bradac, M. Semela and J. Kaiser, Spectrochimica Acta Part B: Atomic Spectroscopy, 2015, 108, 1-7.

30 A. Kramida, Yu. Ralchenko, J. Reader and NIST ASD Team, NIST Atomic Spectra Database (ver. 5.3), [Online]. Available: http://physics.nist.gov/asd [2017, July 18]. National Institute of Standards and Technology, Gaithersburg, MD., 2015.

31 Z. Cermakova, P. Bezdicka, I. Nemec, J. Hradilova, V. Srein, J. Blazek and D. Hradil, Journal of Raman Spectroscopy, 2015, 108, 236-243.

32 I. Alencar, J. Ruiz-Fuertes, K. Schwartz, C. Trautmann, L. Bayarjargal, E. HaussÃijhl and B. Winkler, Journal of Raman spectroscopy, 2016, 47, 978-983.

33 L. A. Haskin, A. Wang, K. M. Rockow, B. L. Jolliff, R. L. Korotev and K. M. Viskupic, Journal of Geophysical Research, 1997, 102, 19293-19306.

\section{Appendix}

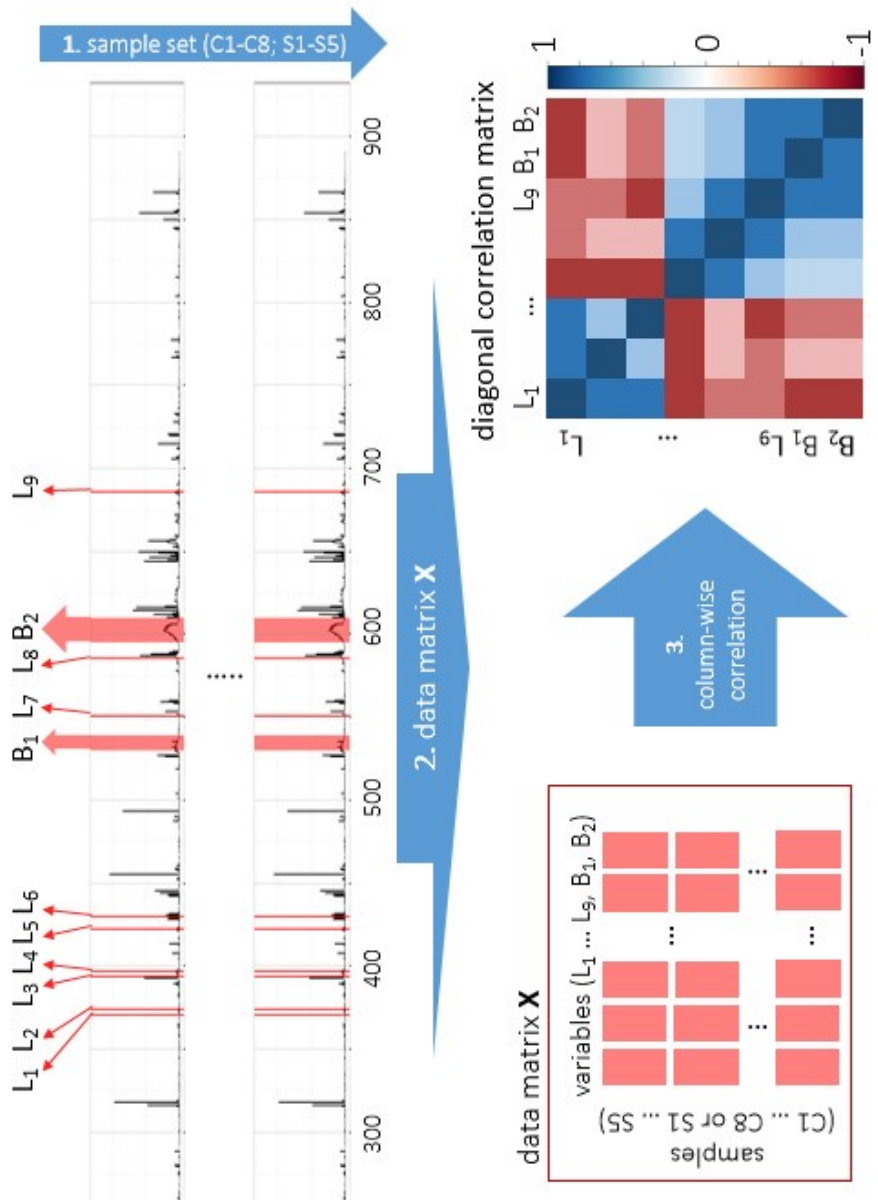

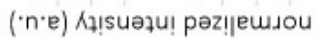

